\title{
The Vienna classification of gastrointestinal epithelial neoplasia
}

R J Schlemper, R H Riddell, Y Kato, F Borchard, H S Cooper, S M Dawsey, M F Dixon, C M Fenoglio-Preiser, J-F Fléjou, K Geboes, T Hattori, T Hirota, M Itabashi, M Iwafuchi, A Iwashita, Y I Kim, T Kirchner, M Klimpfinger, M Koike, G Y Lauwers, K J Lewin, G Oberhuber, F Offner, A B Price, C A Rubio, M Shimizu, T Shimoda, P Sipponen, E Solcia, M Stolte, H Watanabe, H Yamabe

\begin{abstract}
Background-Use of the conventional Western and Japanese classification systems of gastrointestinal epithelial neoplasia results in large differences among pathologists in the diagnosis of oesophageal, gastric, and colorectal neoplastic lesions.
\end{abstract}

Aim-To develop common worldwide terminology for gastrointestinal epithelial neoplasia.

Methods-Thirty one pathologists from 12 countries reviewed 35 gastric, 20 colorectal, and 21 oesophageal biopsy and resection specimens. The extent of diagnostic agreement between those with Western and Japanese viewpoints was assessed by kappa statistics. The pathologists met in Vienna to discuss the results and to develop a new consensus terminology.

Results-The large differences between the conventional Western and Japanese diagnoses were confirmed (percentage of specimens for which there was agreement and kappa values: $37 \%$ and 0.16 for gastric; $45 \%$ and 0.27 for colorectal; and $14 \%$ and 0.01 for oesophageal lesions). There was much better agreement among pathologists $(71 \%$ and 0.55 for gastric; $65 \%$ and 0.47 for colorectal; and $62 \%$ and 0.31 for oesophageal lesions) when the original assessments of the specimens were regrouped into the categories of the proposed Vienna classification of gastrointestinal epithelial neoplasia: (1) negative for neoplasia/dysplasia, (2) indefinite for neoplasia/dysplasia, (3) non-invasive low grade neoplasia (low grade adenoma/ dysplasia), (4) non-invasive high grade neoplasia (high grade adenomal dysplasia, non-invasive carcinoma and suspicion of invasive carcinoma), and (5) invasive neoplasia (intramucosal carcinoma, submucosal carcinoma or beyond).

Conclusion-The differences between Western and Japanese pathologists in the diagnostic classification of gastrointestinal epithelial neoplastic lesions can be resolved largely by adopting the proposed terminology, which is based on cytological and architectural severity and invasion status.

(Gut 2000;47:251-255)
Keywords: early carcinoma; adenoma; dysplasia; oesophagus; stomach; colon

\section{Background}

Large discrepancies between Western and Japanese pathologists have been found in the diagnosis of adenoma/dysplasia versus carcinoma for gastric and colorectal glandular lesions and for oesophageal squamous lesions. ${ }^{1-3}$ These differences in diagnostic criteria have caused considerable problems in the interpretation of Japanese cancer research by Western clinicians and researchers, and vice versa. ${ }^{13}$ These discrepancies therefore called for a united effort to reach a consensus on the nomenclature of gastrointestinal epithelial neoplastic lesions.

Vienna meeting and slide assessments On 5 and 6 September 1998, just before the World Congresses of Gastroenterology, we held a workshop on early neoplasia of the gastrointestinal tract in Vienna, Austria. We invited 48 pathologists from 15 countries to review a circulating slide set and attend this workshop. They were invited from among the most well known and influential pathologists in the field of gastrointestinal neoplasia, and were chosen by the nine participants of a previous meeting in Tokyo in $1996 .^{12}$ Thirty one pathologists from 12 countries agreed to participate and reviewed the slide set, and 27 were able to attend the meeting. The slide set included glass slides of 35 gastric and 20 colorectal lesions, and glass slides plus photomicrographs of 21 oesophageal lesions, for individual review. These slides and photomicrographs were the same histopathological material used in our previous reports, ${ }^{1-3}$ and the results of the pathologists' assessments were similar to those of our previous studies. ${ }^{1-3}$

In short, pathologists with a Western viewpoint made a diagnosis of suspected or definite carcinoma in $17-66 \%$ of the 35 gastric lesions, in $5-40 \%$ of the 20 colorectal lesions, and in $10-67 \%$ of the 21 oesophageal lesions. However, those with a Japanese viewpoint diagnosed suspected or definite carcinoma in $77-94 \%$ of the gastric lesions, in $45-75 \%$ of the colorectal lesions, and in $81-100 \%$ of the oesophageal lesions. When the extent of agreement between the Western and Japanese viewpoints was assessed by kappa statistics, low values representing poor agreement were obtained for the gastric (kappa $=0.16$, agreement in $37 \%$ of ronald-s@fukuoka-u.ac.jp

Accepted for publication 11 January 2000 


\begin{tabular}{|c|c|c|c|c|c|c|c|}
\hline \multirow{2}{*}{\multicolumn{2}{|c|}{ A Stomach }} & \multicolumn{5}{|c|}{$\begin{array}{l}\text { Japanese } \\
\text { viewpoint }\end{array}$} & \multirow[b]{2}{*}{ Tot } \\
\hline & & $R$ & $\mathrm{~L}$ & $\mathrm{H}$ & S & D & \\
\hline \multirow{6}{*}{$\begin{array}{c}\text { Western } \\
\text { viewpoint }\end{array}$} & $R$ & 1 & & & & & 1 \\
\hline & L & & 2 & & 1 & 3 & 6 \\
\hline & $\mathrm{H}$ & & & & 1 & 12 & 13 \\
\hline & S & 1 & & & & 4 & 5 \\
\hline & D & & & & & 10 & 10 \\
\hline & Tot & 2 & 2 & 0 & 2 & 29 & 35 \\
\hline
\end{tabular}

Agreement: $13 / 35=37 \%$

Kappa coefficient: 0.16

(95\% Cl 0.05-0.26)
B Colorectum

\begin{tabular}{cc|ccccc|c} 
& \multicolumn{7}{c}{ viewpoint } \\
& & R & L & H & S & D & Tot \\
\hline \multirow{4}{*}{ Western } & & & & & & 0 \\
viewpoint & & 5 & 2 & 1 & 2 & 10 \\
& L & & & 1 & 1 & 3 & 5 \\
& S & & & & & 2 & 2 \\
& D & & & & & 3 & 3 \\
\hline & Tot & 0 & 5 & 3 & 2 & 10 & 20
\end{tabular}

Agreement: $9 / 20=45 \%$

Kappa coefficient: 0.27

(95\% Cl 0.04-0.49)
C Oesophagus

\begin{tabular}{|c|c|c|c|c|c|c|c|}
\hline & \multicolumn{6}{|c|}{ viewpoint } \\
\hline & & $\mathrm{R}$ & L & $\mathrm{H}$ & $\mathrm{S}$ & D & Tot \\
\hline \multirow{6}{*}{$\begin{array}{l}\text { Western } \\
\text { viewpoint }\end{array}$} & $\mathrm{R}$ & & & & & & 0 \\
\hline & L & & & & 2 & 5 & 7 \\
\hline & $\mathrm{H}$ & & & & & 9 & 9 \\
\hline & $S$ & & & & & 2 & 2 \\
\hline & D & & & & & 3 & 3 \\
\hline & Tot & 0 & 0 & 0 & 2 & 19 & 21 \\
\hline
\end{tabular}

Agreement: $3 / 21=14 \%$

Kappa coefficient: 0.01

(95\% Cl-0.07-0.08)

Figure 1 Extent of agreement between the viewpoints of 31 Western and fapanese pathologists; the diagnoses of (A) 35 gastric, (B) 20 colorectal, and (C) 21 oesophageal lesions are based on their currently used classification systems. $R$, reactive epithelium; L, low grade adenoma/dysplasia; H, high grade adenomaldysplasia; S, suspected carcinoma; D, definite carcinoma; Tot, total. The group of pathologists with a fapanese viewpoint included nine fapanese, three German, one Austrian, and one British pathologist for the gastric diagnoses, nine fapanese, one German, one Austrian, one British, and one Korean pathologist for the colorectal diagnoses, and nine Fapanese pathologists for the oesophageal diagnoses. For each organ system, Western pathologists who diagnosed suspected or definite carcinoma in a manner similar to the nine fapanese specialists in gastrointestinal pathology were considered (and most considered themselves) to have a fapanese viewpoint. For comparisons between Western and fapanese viewpoints the most common opinion was taken as each group's final diagnosis. Values of the kappa coefficient less than 0.4 represent poor agreement, values between 0.4 and 0.75 fair to good agreement, and values exceeding 0.75 excellent agreement.

cases), colorectal (kappa $=0.27$, agreement in $45 \%$ ) and oesophageal (kappa $=0.01$, agreement in $14 \%$ ) slide assessments (fig 1 ).

\section{Current nomenclature}

From our previous studies it was clear that a significant number of the diagnostic differences were in fact due to differences in nomenclature. ${ }^{1-3}$ Western pathologists used the terms adenoma (usually for raised lesions) or dysplasia (for flat or depressed lesions) to indicate the presence of a non-invasive (and therefore still benign) neoplastic epithelial proliferation with the potential to become invasive. A distinction between low grade and high grade dysplasia was conventionally based on such features as whether the neoplastic nuclei were limited primarily to the lower or upper halves of the cells in the glands (or of the epithelium in squamous mucosa). Invasion was mandatory
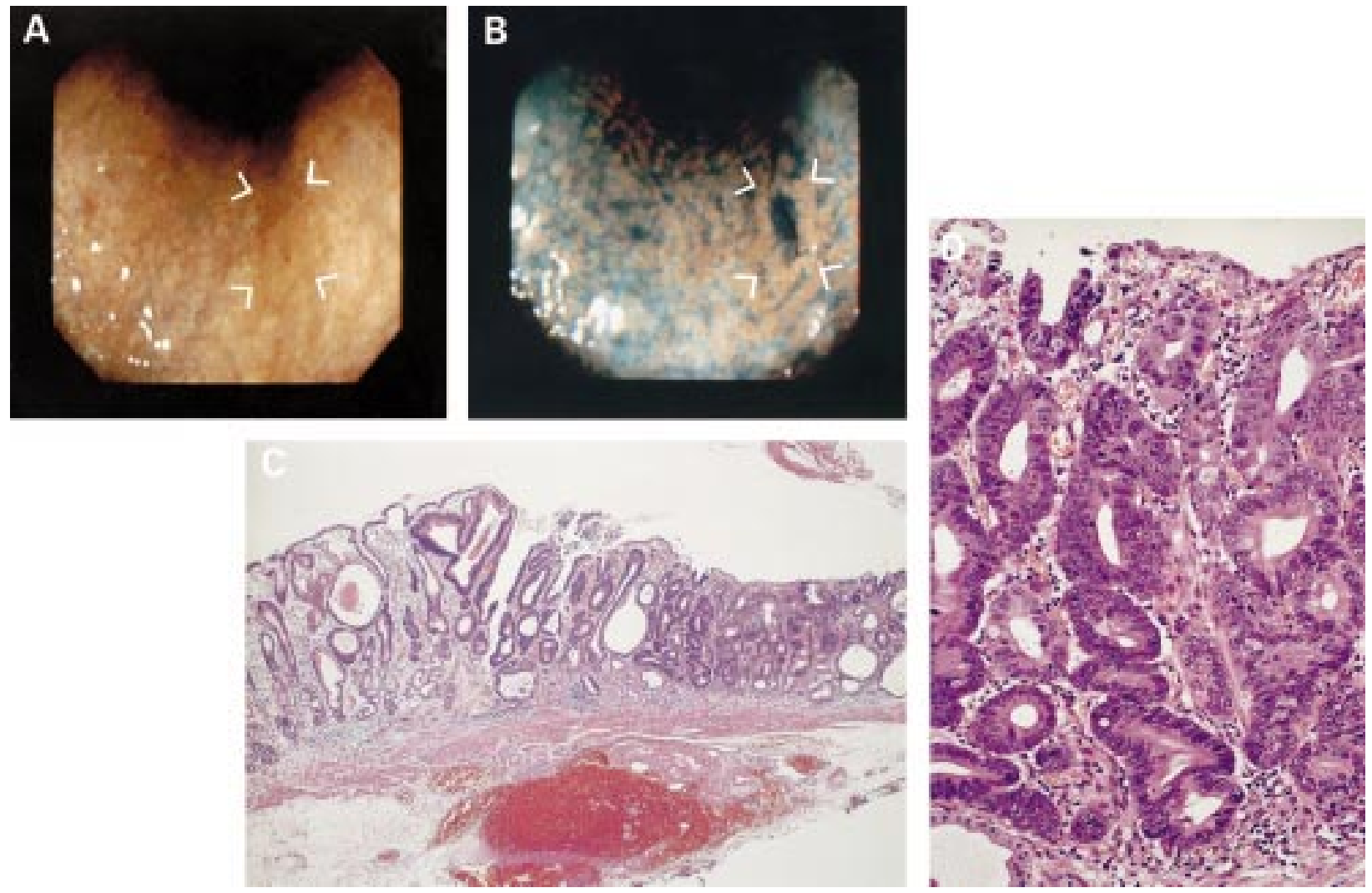

Figure 2 Gastric lesion in a man aged 73 years. (A) Endoscopic picture of a small area of redness (arrowheads) in the corpus amid atrophic gastric mucosa. (B) Endoscopic picture after spraying indigo carmine, revealing a shallow depressed lesion (arrowheads). (C) Endoscopically resected specimen of this lesion that was diagnosed as high grade adenomaldysplasia by most Western pathologists and as carcinoma (non-invasive carcinoma) by most Fapanese pathologists. (D) Details of (C), showing budding of glands and a trabecular growth pattern that made several Western and Fapanese pathologists suspect invasion into the lamina propria. 
for a Western diagnosis of carcinoma and was defined as being present when the lamina propria was involved in the stomach or oesophagus and when the submucosa was involved in the colon or rectum. In contrast, it was clear that for Japanese pathologists the diagnosis of carcinoma was based on cytological changes (variably sized and enlarged nuclei, rounded nuclei, loss of polarity, prominent nucleoli) and architectural changes (complex budding or branching of glands, back to back glands). This led the Japanese to use the term "mucosal carcinoma" without making a distinction as to whether or not there was invasion into the lamina propria. ${ }^{2}$

\section{Discussion of differences}

As a consequence of the different nomenclature, there were many early neoplastic lesions that Western pathologists diagnosed as high grade adenoma/dysplasia and Japanese pathologists diagnosed as (non-invasive) carcinoma (fig 1). In addition, a different interpretation of nuclear features accounted for several lesions being diagnosed as low grade adenoma/ dysplasia by Western pathologists and as carcinoma by the Japanese (fig 1). To overcome these differences and to reach a consensus on a new classification of epithelial neoplasia, a better understanding of the current Western and Japanese terminology was needed. At the workshop we therefore discussed many of the circulated lesions thoroughly. It became evident that a classification of epithelial neoplasia should include grading based on the aspect of invasion, as invasion is an indicator of metastatic potential and therefore of major prognostic significance. Moreover, all pathologists agreed that a classification should include the term "indefinite for dysplasia", to be used when one cannot decide whether a lesion is non-neoplastic or neoplastic, and the term "suspicion of invasive carcinoma" for when one finds it hard to determine whether or not invasion into the lamina propria is present.

Invasion may be hard to define. For lesions such as that depicted in fig 2 , many Western pathologists found it difficult to decide between a diagnosis of high grade adenoma/ dysplasia and suspected carcinoma, while many Japanese pathologists diagnosed noninvasive carcinoma but also suspected that invasion into the lamina propria might already be present. Indeed, we found that in many cases neither Western nor Japanese pathologists could distinguish between "high grade adenoma/dysplasia", "non-invasive carcinoma (carcinoma in situ)" and "suspicion of invasive carcinoma" in a reproducible way. While for research purposes it might be useful to distinguish between these three terms, from a therapeutic viewpoint such a distinction might not be essential as in each of these cases local excision, either endoscopically or surgically, would be advisable.

\section{Consensus classification}

For these reasons the pathologists attending the workshop developed the classification shown in table 1. Epithelial neoplastic lesions
Table 1 Vienna classification of gastrointestinal epithelial neoplasia

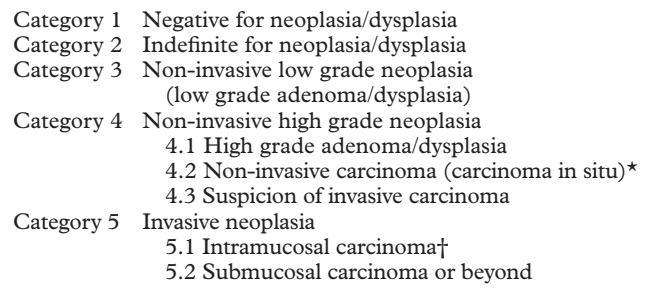

*Non-invasive indicates absence of evident invasion.

+Intramucosal indicates invasion into the lamina propria or muscularis mucosae.

are classified into five categories, some of which have subcategories. This classification is meant to be applied throughout the entire gastrointestinal tract and for the diagnosis of biopsy as well as resected specimens. The division into five categories is especially helpful for clinicians in their decision about what to do with a biopsy result, as the grading reflects differences in expected biological behaviour of the lesions.

For biopsies diagnosed as category 1 -negative for neoplasia/dysplasia (including normal, reactive, regenerative, hyperplastic, atrophic, and metaplastic epithelium) - further follow up of the lesion may or may not be necessary, as clinically indicated. In the case of category 2-indefinite for neoplasia/ dysplasia-follow up is needed because of uncertainty about the real nature of the lesion. In category 3-non-invasive low grade neoplasia (low grade adenoma/dysplasia) — neoplasia is present but the risk of developing invasive carcinoma is low. Clinicians may consider local treatment of the lesion or opt for follow up. In category 4-non-invasive high grade neoplasia - the risk of invasion and development of metastases is increased. Local treatment such as endoscopic mucosal resection or local surgical treatment would be indicated. In the case of category 5-invasive neoplasia- the risk of subsequent deeper invasion and metastases is so high that treatment is urgently needed and should only be withheld in cases with clinical contraindications. In general, before a decision on treatment is made, one should always take into account the possibility of sampling error which may lead to underestimation of the grade of neoplastic change or depth of invasion. The histological diagnosis of biopsy specimens is only part of the total clinical information and should be supplemented by available endoscopic, radiological, and ultrasonographic assessments of the depth of invasion.

An important feature of this classification is the grouping into one category of 4.1 high grade adenoma/dysplasia, 4.2 non-invasive carcinoma (CIS), and 4.3 suspicion of invasive carcinoma. For research purposes, the subdivision of category 4 will still be important to clarify if meaningful differences exist among these subcategories but for clinical purposes classification into the five major categories should suffice. Figure 3 shows the original assessments of the slide set read by the 31 pathologists prior to the workshop regrouped 


\begin{tabular}{|c|c|c|c|c|c|c|c|}
\hline \multirow{2}{*}{\multicolumn{2}{|c|}{ A Stomach }} & \multicolumn{5}{|c|}{$\begin{array}{l}\text { Japanese } \\
\text { viewpoint }\end{array}$} & \multirow[b]{2}{*}{ Tot } \\
\hline & & $\mathrm{C} 1$ & $\mathrm{C} 2$ & $\mathrm{C} 3$ & $\mathrm{C} 4$ & $\mathrm{C} 5$ & \\
\hline & $\mathrm{C} 1$ & 1 & & & & & 1 \\
\hline $\begin{array}{l}\text { Western } \\
\text { viewpoint }\end{array}$ & $\begin{array}{l}\text { C2 } \\
\text { C3 }\end{array}$ & & & 2 & 4 & & 6 \\
\hline & $\mathrm{C} 4$ & 1 & & & 12 & 5 & 18 \\
\hline & C5 & & & & & 10 & 10 \\
\hline & Tot & 2 & 0 & 2 & 16 & 15 & 35 \\
\hline
\end{tabular}

Agreement: $25 / 35=71 \%$

Kappa coefficient: 0.55

(95\% Cl 0.32-0.77)
B Colorectum

\begin{tabular}{|c|c|c|c|c|c|c|c|}
\hline & \multicolumn{6}{|c|}{$\begin{array}{l}\text { Japanese } \\
\text { viewpoint }\end{array}$} \\
\hline & & $\mathrm{C} 1$ & $\mathrm{C} 2$ & C3 & $\mathrm{C} 4$ & C5 & Tot \\
\hline \multirow{6}{*}{$\begin{array}{l}\text { Western } \\
\text { viewpoint }\end{array}$} & C1 & & & \multirow{5}{*}{5} & \multirow{5}{*}{5} & \multirow{5}{*}{2} & 0 \\
\hline & $\mathrm{C} 2$ & & & & & & 0 \\
\hline & C3 & & & & & & 10 \\
\hline & C4 & & & & & & 7 \\
\hline & $\mathrm{C} 5$ & & & & & & 3 \\
\hline & Tot & 0 & 0 & 5 & 10 & 5 & 20 \\
\hline & & $\begin{array}{l}\text { gree } \\
\text { Kapp }\end{array}$ & $\mathrm{me}$ & fffi & 20 & & \\
\hline
\end{tabular}

(95\% Cl 0.18-0.76)

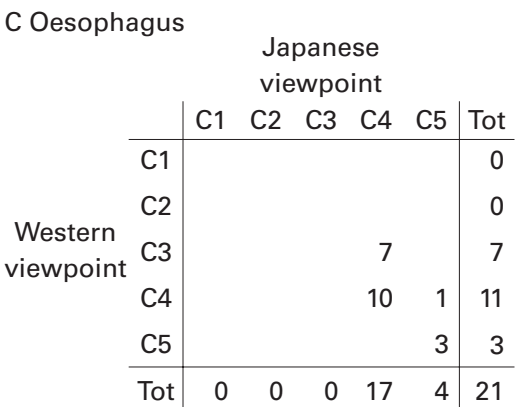

Agreement: $13 / 21=62 \%$ Kappa coefficient: 0.31 (95\% Cl 0.08-0.53)

Figure 3 Extent of agreement between the viewpoints of 31 Western and fapanese pathologists; the diagnoses for (A) 35 gastric, (B) 20 colorectal, and (C) 21 oesophageal lesions are based on the five categories of the Vienna classification (table 1). C1, negative for neoplasialdysplasia; C2, indefinite for neoplasialdysplasia; C3, non-invasive low grade neoplasia; C4, non-invasive high grade neoplasia; C5, invasive neoplasia; Tot, total. For details on the comparisons between Western and fapanese viewpoints see the legend to fig 1.

into these five categories. From this analysis it is clear that without any additional education of the pathologists on the use of this classification, the mere fact that high grade adenoma/dysplasia was grouped with noninvasive and suspicion of invasive carcinoma led to much better agreement between Western and Japanese pathologists for gastric lesions (increase in kappa from 0.16 to 0.55 , increased agreement from $37 \%$ to $71 \%$ of cases), colorectal lesions (increase in kappa from 0.27 to 0.47 , increased agreement from $45 \%$ to $65 \%$ ), and oesophageal lesions (in- crease in kappa from 0.01 to 0.31 , increased agreement from $14 \%$ to $62 \%$ ).

Agreement between Western and Japanese pathologists would be further improved if there were a better consensus on how to distinguish low grade from high grade non-invasive neoplasia. This would avoid the discrepancy caused by lesions being diagnosed as category 3 by Western pathologists but as category 4 by Japanese pathologists (fig 3). This problem is mainly the result of different interpretations of nuclear features. Western pathologists tend to put more weight on the degree of nuclear
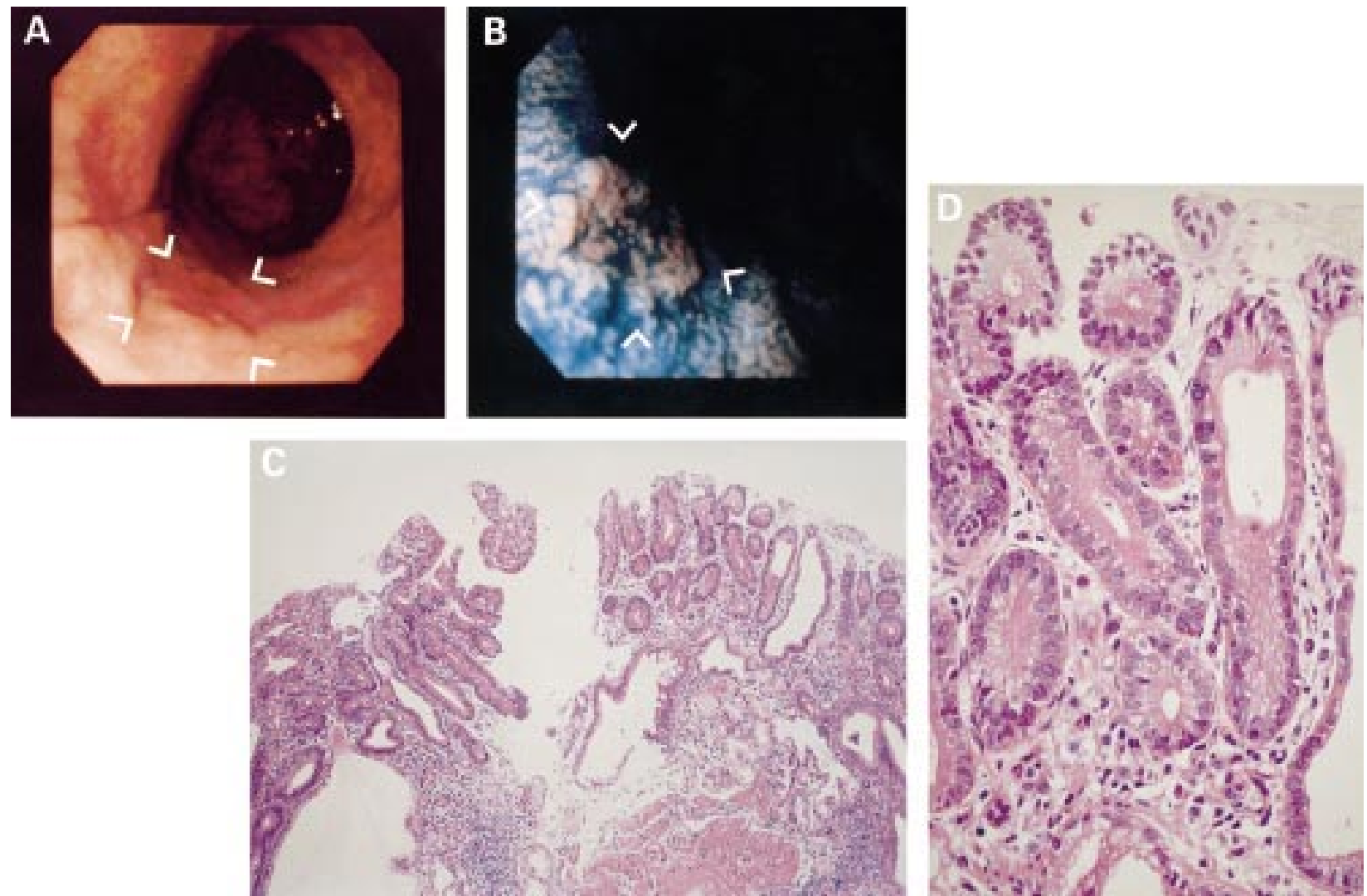

Figure 4 Gastric lesion in a man aged 67 years. (A) Endoscopic picture of a slightly elevated lesion with a central depression (arrowheads) in the corpus. (B) Endoscopic picture after spraying indigo carmine, revealing the borders of the lesion more clearly (arrowheads). (C) Biopsy specimen of this lesion that was diagnosed as low grade adenomaldysplasia by most Western pathologists and as carcinoma (non-invasive carcinoma) by most fapanese pathologists a few months before the meeting, but as category 4 (see classification in table 1) at the end of the meeting. (D) Details of (C), showing mild stratification but enlarged, rounded nuclei with prominent nucleoli. 
stratification while Japanese pathologists attach more importance to the variable size and roundness of the nuclei and the prominence of the nucleoli. That this problem may be overcome was suggested by a quick test of the proposed classification at the end of the workshop. Having become more familiar with each other's diagnostic criteria and having reached a consensus on the classification, the Western and Japanese pathologists were shown photomicrographs of several of the cases from the circulated slide set that had not yet been discussed and were asked to classify them into the five categories detailed in table 1 . Shown the biopsy specimen depicted in fig 4 , all of the pathologists indicated that it was a category 4 lesion. This agreement was much greater than that of the diagnoses returned a few months earlier. At that time the same biopsy had been diagnosed by the majority of the Western pathologists as low grade adenoma/dysplasia and by the majority of the Japanese pathologists as non-invasive carcinoma.

\section{Conclusion}

In summary, this new classification is practical and should be useful for resolving many of the discrepancies between Western and Japanese pathologists in the diagnosis of gastrointestinal epithelial neoplastic lesions. Such a resolution should contribute to better communication between pathologists and clinicians and to a better understanding of research data in the fields of gastroenterology, epidemiology, and molecular biology. This consensus classification is currently undergoing further evaluation and refinement. In particular, the task of defining histological criteria for separating the five categories will be taken on by a series of working groups set up for all organs in the gastrointestinal tract, and comments are being sought from additional pathologists and clinicians. To avoid further confusion concerning the terms adenoma, dysplasia, and carcinoma, we would suggest that all authors indicate the categories of this consensus classification in addition to their usual diagnoses when they report in the international literature.

Authors' affiliations

R J Schlemper, Department of Internal Medicine, Fukuoka University School of Medicine, Fukuoka, Japan; R H Riddell, Department of Pathology and Molecular Medicine, McMaster University Medical Centre, Hamilton, Ontario, Canada; Y Kato, Depart- ment of Pathology, Cancer Institute, Tokyo, Japan; F Borchard, Institute of Pathology, Klinikum Aschaffenburg, Aschaffenburg, Germany; H S Cooper, Department of Pathology, Fox Chase Cancer Center, Philadelphia, Pennsylvania, USA; S M Dawsey, Division of Clinical Sciences, National Cancer Institute, Bethesda, Maryland, USA; M F Dixon, Department of Histopathology, Centre for Digestive Diseases, University of Leeds, Leeds, UK; C M Fenoglio-Preiser, Department of Pathology and Laboratory Medicine, University of Cincinnati Medical Center, Cincinnati, Ohio, USA; J-F Fléjou, Service of Anatomical Pathology, Saint-Antoine Hospital, Paris, France; K Geboes, Department of Pathology, University Hospital KUL, Leuven, Belgium; T Hattori, Department of Pathology, Shiga University of Medical Science, Otsu, Japan; T Hirota, Special Reference Laboratory, Tokyo, Japan; M Itabashi, Department of Pathology, Ibaraki Prefectural Central Hospital, Tomobe, Japan; M Iwafuchi, Department of Medical Technology, Niigata University, Niigata, Japan; A Iwashita, Department of Pathology, Fukuoka University Chikushi Hospital, Fukuoka, Japan; Y I Kim, Department of Pathology, Seoul National University, Seoul, Korea; T Kirchner, Institute of Pathology, University Erlangen-Nürnberg, Erlangen, Germany; M Klimpfinger, Institute of Pathology and Microbiology, Kaiser Franz Josef Spital, Vienna, Austria; M Koike, Department of Pathology, Tokyo Metropolitan Komagome Hospital, Tokyo, Japan; G Y Lauwers, Department of Pathology, University of Florida HSC, Gainesville, Florida, USA; K J Lewin, Department of Pathology and Laboratory Medicine, UCLA Center for Health Sciences, Los Angeles, California, USA; G Oberhuber, Department of Clinical Pathology, University of Vienna, Vienna, Austria; F Offner, Department of Pathology, University of Innsbruck, Innsbruck, Austria; A B Price, Department of Pathology, Northwick Park Hospital and St Marks Hospitals, Middlesex, UK; C A Rubio, Gastrointestinal and Liver Pathology Research Laboratory, Karolinska Institute, Stockholm, Sweden; M Shimizu, Department of Surgical Pathology, Hokkaido University Hospital, Sapporo, Japan; T Shimoda, Clinical Laboratory Division, National Cancer Center Hospital, Tokyo, Japan; P Sipponen, Department of Pathology, Jorvi Hospital, Espoo, Finland; E Solcia, Department of Human Pathology, University of Pavia and Policlinico San Matteo, Pavia, Italy; M Stolte, Institute of Pathology, Klinikum Bayreuth, Bayreuth, Germany; H Watanabe, First Department of Pathology, Niigata University School of Medicine, Niigata, Japan; $\mathrm{H}$ Yamabe, Laboratory of Anatomic Pathology, Kyoto University Hospital, Kyoto, Japan.

1 Schlemper RJ, Itabashi M, Kato Y, et al. Differences in diagnostic criteria for gastric carcinoma between Japanese and Western pathologists. Lancet 1997;349:1725-9.

2 Schlemper RJ, Itabashi M, Kato Y, et al. Differences in the diagnostic criteria used by Japanese and Western pathologists to diagnose colorectal carcinoma. Cancer 1998;82:60gists

3 Schlemper RJ, Dawsey SM, Itabashi M, et al. Differences in diagnostic criteria for esophageal squamous cell carcinoma between Japanese and Western pathologists. Cancer 2000; 88:996-1006. 